\title{
Reflex anal dilatation: effect of parting the buttocks on anal function in normal subjects and patients with anorectal and spinal disease
}

\author{
N W Read, W M Sun
}

\begin{abstract}
Anal dilatation in response to gentle parting of the buttocks has been advocated as a sign of sexual abuse in children, but nothing is known of the physiology of this response or its existence in normal subjects, in patients with spinal disease, and in patients with a weak sphincter and whether it can be elicited after training. To answer these questions we investigated the effect of parting the buttocks on anal function. Combined anal manometry and electromyography was conducted in six normal subjects (five men, one woman, aged 19-53 years), in 18 patients with faecal incontinence (three men, 15 women, aged 30-80 years), and in seven paraplegic patients (six men, one woman, aged 25-36 years), in four of whom the posterior sacral roots had been cut. Parting the buttocks in normal subjects reduced the pressure in the anal canal from 102 (20) to 14 (3) $\mathrm{cm} \mathrm{H}_{2} \mathrm{O}$ (mean (SEM), $\mathrm{p}<0.00001$ ), but did not cause the anus to gape. This drop in pressure was associated with increased electrical activity in the external anal sphincter. Normal subjects could consciously relax the external anal sphincter and reduce the anal pressure but not so as to result in anal gaping during traction on the buttocks, even after anal dilatation. Stimulation of the anal lining by moving a probe in and out of the anal canal increased the activity of the external anal sphincter, raising anal pressures. Paraplegic patients who had lost conscious control of their external sphincters showed anal gaping when the buttocks were parted. A similar phenomenon was seen in patients with faecal incontinence who had weakness of the external anal sphincter, while incontinent patients with weakness of both sphincters showed anal gaping even at rest. Inasmuch as the results of our study can be applied to children, the data suggest that reflex anal dilatation should only be used to support a diagnosis of sexual abuse if sphincter function is otherwise normal and there is no evidence of cerebrospinal disease. Although our results do not support the notion that children could become so conditioned to repeated digital or penile penetration of the anus that they can cause the anus to gape when the buttocks are parted, neither do they exclude it.
\end{abstract}

Unit

Unit, Centre for Huma

General Hospital

Sheffield S5 7AU

N W Read

W M Sun

Correspondence to:

Professor N W Read.

Accepted for publication

13 August 1990
The use of 'reflex' anal dilatation as a clinical sign of sexual abuse in children ${ }^{12}$ is controversial and has caused great public concern in recent years. ${ }^{3-8}$ The sign is elicited by a gentle but firm parting of the buttocks, whereupon the sphincter is said to gape so that a clear view of the rectal mucosa is obtained. No physiological investigation of the effect of parting the buttocks on anorectal function has ever been carried out on normal subjects or patients with well defined anal or neurological disease. Such studies are essential if the sign is to be correctly interpreted. Ethical constraints and the politically sensitive nature of the test precluded such a study in children. Thus we studied the effect of parting the buttocks on anal function in normal adult subjects and incontinent and spinal patients using multiport anal manometry and electromyography. The purpose of our investigation was to answer the following questions. Does gentle traction on the buttocks cause anal relaxation in normal subjects, and if not, can it be elicited after a period of 'training' by mechanical dilatation of the anus or during stimulation of the anal epithelium? Is the sign present in patients with weak external or internal anal sphincters? Is the sign present in patients with paraplegia, and is it affected by abolition of the spinal reflexes section of the dorsal nerve roots? We believe that our results elucidate the pathophysiology of this sign and help to put its use in child sexual abuse in a more correct perspective.

\section{Methods}

SUBJECTS

Studies were carried out on six normal adult volunteers (five men, one woman; aged 19-53 years). None had had any disturbances in anorectal function or had had anal disease or surgery. They were all asked if they had had experienced passive anal intercourse and all denied it. None had previously undergone a digital or instrument examination of the rectum. Each subject gave written informed consent for the study to be conducted, but they were not informed of the true reason for carrying out the study until it was completed. The protocol was approved by the Ethical Subcommittee of the Sheffield Area Health Authority.

Parting the buttocks was also carried out in 18 patients who had been referred to our unit complaining of faecal incontinence $(\mathrm{M}: \mathrm{F}=3: 15$; aged $30-80$ years) and in seven paraplegic patients $(M: F=6: 1$; aged $25-36$ years) with the level above $T 1$, in four of whom the posterior sacral roots had been cut. ${ }^{9}$ The manometric data obtained from the patient groups were compared with those from 25 volunteers approximately matched for age and sex $(M: F=5: 20$; aged 21-63 years), and the significance of the data was assessed by Student's $t$ test. 
PROTOCOL IN NORMAL SUBJECTS

With the subject lying in the left lateral position a five lumen manometric probe of $0.4 \mathrm{~cm}$ diameter was inserted into the anus with perfused side holes situated at $0 \cdot 5,1 \cdot 0,1 \cdot 5,2 \cdot 0$, and $2.5 \mathrm{~cm}$ from the anal verge. A bipolar wire electrode was inserted into the external anal sphincter. ${ }^{10}$ The resting pressure and external anal sphincter electrical activity were recorded for over 15 minutes during which they gradually fell to establish a steady baseline.$^{10}$ The buttocks were then parted by laying the thumbs approximately $1.5 \mathrm{~cm}$ lateral to the anal margin and firmly but gently pulling them apart with the fingers around the outer aspects of the buttocks, maintaining the tension for a timed half a minute. This manoeuvre was repeated on two further occasions with a one minute interval between each test. Next the subject was asked to consciously attempt to relax the sphincter while the buttocks were parted and the manoeuvre was repeated on two more occasions.

After a rest of five minutes the small flexible anal probe was removed and replaced with a larger probe of either $1.5,2.0$, or $2.5 \mathrm{~cm}$ in diameter (the maximum that subjects could

Channel $\mathrm{cm} \mathrm{H}_{2} \mathrm{O}$

1

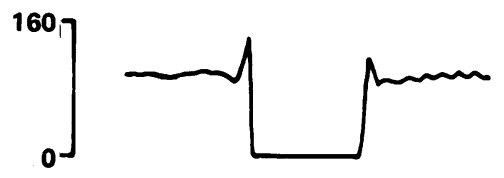

2

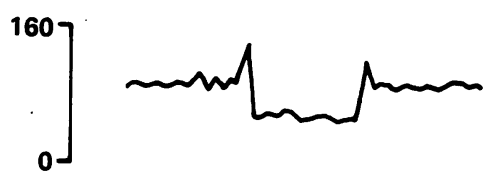

3

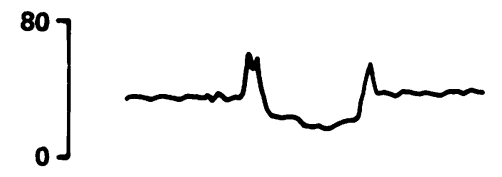

4

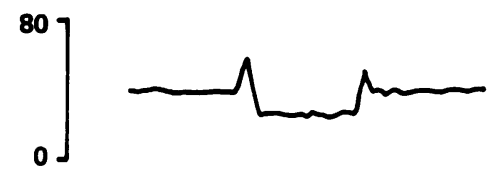

5

1: Recordings of ana pressures and the electrical activity of the external anal sphincter in a typical normal subject. Channels 1-5 represent ports situated in the anal canal $0 \cdot 5,1 \cdot 0,1 \cdot 5$ 2.0 , and $2.5 \mathrm{~cm}$ from the anal verge. Note that the anal pressures fell appreciably when the buttocks were parted. This was associated with the increased electrical activity of the external anal sphincter, particularly when the buttocks were first parted and when they were released. tolerate without pain), with four manometric channels situated at each quadrant of the probe. This was inserted into the anal canal so that the manometric channels were situated at the zone of the highest pressure for half a minute. The basal sphincter pressure was recorded. Then subjects were asked to relax the external sphincter and the pressure was recorded for a further minute. The probe was removed and reinserted on two further occasions with a pause of a minute between each. The large probe was then removed, the small probe was reinserted, and after an interval of five minutes the buttocks were parted again as indicated above to determine whether a period of anal dilatation had altered the responses. Finally, in order to determine whether mechanical stimulation of the anal canal could cause anal relaxation, the small manometric probe was moved $10 \mathrm{~cm}$ backwards and forwards in the anal canal, five times in 15 seconds, returning to its original position. The pressure recorded immediately after movement of the probe was compared with that recorded immediately before.

\section{PROTOCOL IN PATIENTS}

Paraplegic patients and patients with faecal incontinence but no spinal disease were assessed by placing them on their side in the knee-elbow position and first observing the perineum to determine whether the anus was gaping before inserting the manometric probe. Secondly, the buttocks were parted as indicated above, and the characteristics and timing of any dilatation were noted. Thirdly, a routine manometric/electrophysiological study was performed, as described previously. ${ }^{10}$

\section{Results}

\section{RESULTS OBTAINED FROM NORMAL SUBJECTS}

\section{Effect of parting the buttocks}

Parting the buttocks did not cause the anus to gape in any normal subject, but it did reduce the highest anal pressure in every subject from 102 (20) to 14 (3) $\mathrm{cm} \mathrm{H}_{2} \mathrm{O}$ (mean (SEM); $\mathrm{p}<0.00001$ ). This drop was associated with increased electrical activity in the external anal sphincter for as long as the force was applied, with bursts of activity when the buttocks were first parted and when they were released (Fig 1). The anal pressure recorded after the buttocks had been parted for 30 seconds (80 (19) $\mathrm{cm} \mathrm{H}_{2} \mathrm{O}$ ) was significantly lower than the basal pressure $(\mathrm{p}<0.02)$.

When subjects were asked to consciously relax their sphincters the basal pressure fell from 102 (20) to $\left(96(9) \mathrm{cm} \mathrm{H}_{2} \mathrm{O}\right)(\mathrm{p}<0.05)$, but the pressures achieved when parting the buttocks ( $15(5) \mathrm{cm} \mathrm{H}_{2} \mathrm{O}$ ) were no lower than those recorded when subjects were not instructed to relax the sphincter and the anus did not gape.

Anal distension with the large diameter probe for half a minute reduced the basal anal pressures to $86\left(12 \mathrm{~cm} \mathrm{H}_{2} \mathrm{O}(\mathrm{p}<0.001)\right.$ but the pressures recorded while parting the buttocks $(15$ (4) $\mathrm{cm}$ 
$\mathrm{H}_{2} \mathrm{O}$ ) were still similar to those recorded before anal distension and the anus did not gape.

\section{Anal pressures recorded using the large probe}

Two subjects were tested using the $1.5 \mathrm{~cm}$ diameter probe, three using the $2 \mathrm{~cm}$ probe, and one using the $2.5 \mathrm{~cm}$ probe. Although pressures tended to be higher in the posterior aspect of the sphincter, there were no significant differences between the radial pressures.

When the subjects were instructed to consciously relax the external sphincter with the large probe in situ the anal pressures declined from 137 (8) to 93 (6) $\mathrm{cm} \mathrm{H}_{2} \mathrm{O}$ ( $\mathrm{p}<0.0001$ ). This reduction in pressure was associated with a reduction in external sphincter activity.

Stimulation of the anal canal by moving the probe Moving a probe in and out of the anal canal elicited electrical activity in the external anal sphincter. The pressures immediately after this manoeuvre were significantly higher than the pressures immediately before it (104 (23) $v 80$ (20) $\mathrm{cm} \mathrm{H}_{2} \mathrm{O} ; \mathrm{p}<0.05$ ).

\section{RESULTS OBTAINED FROM PATIENTS}

Results obtained from patients varied according to the pathophysiological abnormality.

\section{Paraplegic patients}

Four paraplegic patients whose posterior sacral roots had been cut, preventing low spinal

Figure 2: A diagram showing the pressures recorded $(A)$ under resting conditions and $(B)$ during maximum contraction (squeeze) of the anal sphincter and $(C)$ the duration of the electrical activity of the external ana sphincter in response to recta sphincter in response to rectal
distension with $100 \mathrm{ml}$ of air in incontinent patients compared with normal subjects. The box indicates the normal range;

= patients who showed no anal gaping, $\square=$ those who showed anal gaping only when the buttocks were parted, and $\mathbf{\Delta}=$ those who showed anal gaping under resting conditions.

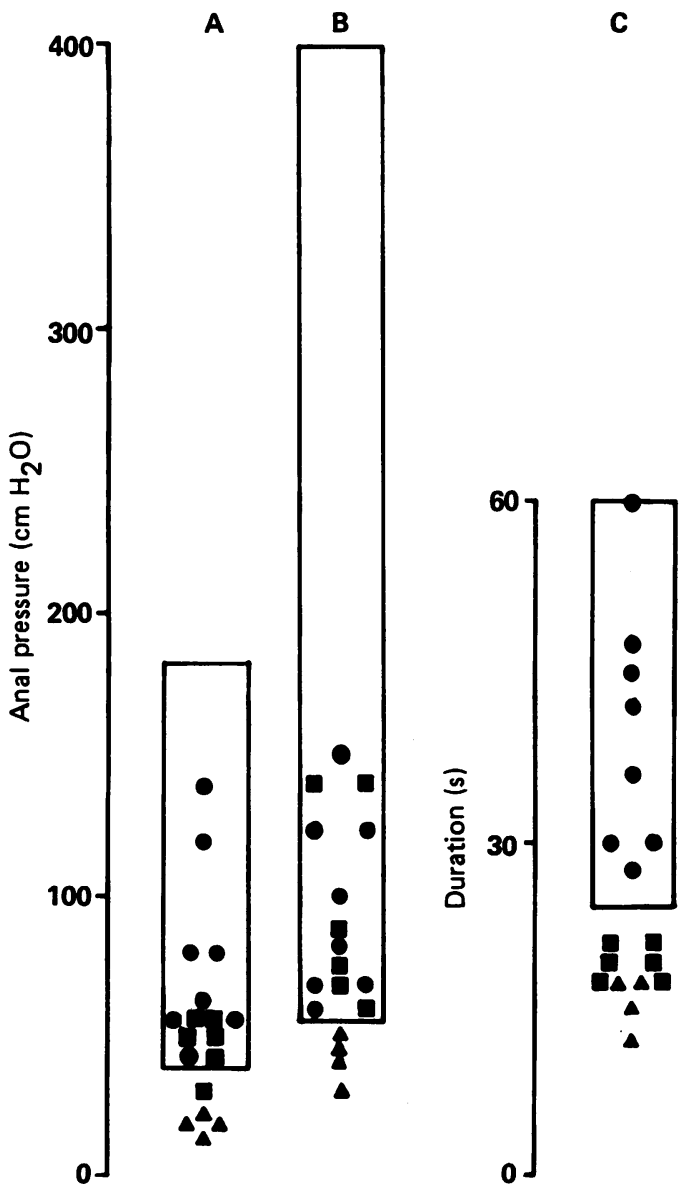

reflexes, did not show anal gaping at rest, but the anus gaped immediately the buttocks were parted and remained open for the duration of the manoeuvre. Perineal stimulation or rectal distension in these patients did not elicit increases in electrical activity of the external anal sphincter.

Three paraplegic patients whose posterior roots had not been cut reacted differently. The anus was closed at rest and contracted strongly when the buttocks were parted. This contraction only lasted for less than five seconds whereupon the anus gaped. The electrical record showed only brief increases in external anal sphincter activity during perineal scratching and rectal distension.

In all seven spinal patients the anus remained wide open for at least five seconds after the tension had been removed.

\section{Incontinent patients with no spinal lesion}

Ten out of 18 patients showed anal gaping. Four female patients had an anal gap at rest of about $1 \mathrm{~cm}$ through which the observer could look into the rectum. Once the buttocks were parted the anus relaxed completely and the rectum could be seen at least $5 \mathrm{~cm}$ above the anorectal ring. The wide dilatation of the anus lasted for at least two seconds after the parting force had stopped. With manometric testing these patients had abnormally low basal and squeeze pressures, no anal relaxation during rectal distension, and an abnormally brief external anal sphincter response to rectal distension, suggesting weakness of both internal and external anal sphincters (Fig 2).

Five female patients and one male patient had anuses that were closed at rest and gaped widely when the buttocks were parted but recovered as soon as the force was removed. These patients had normal basal and squeeze pressure, but the duration of the external anal sphincter response to rectal distension was abnormally brief (Fig 2). These features suggested impaired reflex responses of the external anal sphincter.

None of the remaining eight patients showed anal gaping when the buttocks were parted. Their resting anal pressures and squeeze pressures and the duration of the electrical activity of the external anal sphincter during rectal distension were all within the normal range (Fig 2).

\section{Discussion}

The results of this study show that firm but gentle parting of the buttocks can reduce the anal pressure in the outermost aspect of the sphincter in normal adult subjects. This drop in anal pressure, however, is normally compensated by an increase in the activity of the external anal sphincter which remains raised above baseline values as long as the force is applied on the buttocks. These results suggest that in normal subjects external sphincter activity prevents the anus relaxing and gaping.

The importance of the external sphincter in preventing anal gaping is emphasised by our observations in patients. Traction applied to the sphincter by parting the buttocks of paraplegic 
patients, whose posterior sacral roots had been cut to abolish spinal reflexes, caused no increased activity in the external sphincter and a slow relaxation of the unsupported internal sphincter. This suggests that the external sphincter response is a spinal reflex whereas the slow dilatation is either an enteric nervous or myogenic response to stretching the unsupported sphincter. Similarly, anal gaping on traction occurs only in those incontinent patients who show an attenuated external sphincter response to rectal distension, while gaping is present even at rest in incontinent patients with abnormally weak internal and external sphincters. While showing the role of the external sphincter in preventing anal dilatation, they also show that the internal sphincter can maintain anal closure at rest by its own myogenic properties but can be made to relax by traction on the buttocks as well as by rectal distension. Perhaps during defecation the stretching of the perineum produced by squatting normally acts together with faecal distension of the rectum to induce internal sphincter relaxation. Is such anal dilatation a reflex or is it merely the result of mechanical traction?

The transient nature of the external sphincter responses to parting the buttocks and to rectal distension in paraplegic patients who have not had their dorsal roots cut suggests that spinal reflexes are heavily modulated by cerebral mechanisms in normal subjects. ${ }^{12} 13$

Modulation by cerebral influences raises the question: To what extent can the external sphincter be trained to relax in response to traction on the buttocks? We know that the reflex external anal sphincter contraction induced by rectal distension and possibly by perineal stretching must be inhibited during the act of defecation. Although the results of the present study confirmed that normal subjects could relax the sphincter at will, ${ }^{14}$ contraction of the external sphincter still occurred during traction, and the anus did not gape. Even a period of anal dilatation did not facilitate gaping.

It was impossible to carry out this study in children for ethical reasons, and so our data must be treated with caution. Nevertheless, our results offer no support for the contention that people who had been sexually abused could have trained themselves to relax the sphincter in response to repetitive penetration of the anus with a finger or penis. On the contrary, the movement back and forth of an anal probe will increase external sphincter activity and sphincter pressures. It is important to emphasise, however, that our observations were made in adults in the laboratory after a brief period of training and do not exclude the possibility that more prolonged conditioning, carried out on children in a less experimental setting, could lead to inhibition of the external anal sphincter and result in anal gaping.

Our data, however, do indicate that reflex anal dilatation can occur in patients with weak external anal sphincters or disease of the central nervous system, or both. Although it is possible that sexual abuse could have damaged the sphincter, the sign can only reliably be used to support a diagnosis of sexual abuse if sphincter function is otherwise normal, the subjects have no evidence of cerebrospinal disease, and there are other stigmata of abuse.

1 Hobbs CJ, Wynne JM. Buggery in childhood - a common syndrome of child abuse. Lancet 1986; ii: 792-6.

2 Wright CM, Fraser EM, Denman M, Duke L. Detection of sexual abuse in children. Lancet 1987; ii: 218 .

3 Hey F, Buchan PC, Littlewood JM, Hall RI. Differential diagnosis in child sexual abuse. Lancet 1987; i: 283.

4 Clayden G. Anal appearances and child abuse. Lancet 1987; i 620.

5 Zeitlin $\mathrm{H}$. Investigation of the sexually abused child. Lance 1987; ii: 842-5.

6 Northern Regional Health Authority. Child sexual abuse principles of good practice. $\operatorname{Br} \mathcal{F}$ Hosp Med 1988; 39: 54-5, $57-62$.

7 Anonymous. Cleveland, England, child abuse in the public eye [Editorial]. Am f Dis Child 1989; 143: 651-2.

8 Annnymous. Child abuse after Cleveland [Editorial]. Lancet 1988; ii: $139-40$.

9 Brindly GB. The actions of parasympathetic and sympathetic nerves in human micturition, erection, and seminal emmis sion, and their restoration in paraplegic patients by implanted electrical stimulators. Proc $R$ Soc Lond 1988; B235 $111-20$

10 Sun WM, Read NW. Anorectal function in normal human subjects: effect of gender. Int $\mathcal{F}$ Colorect Dis 1989; 4: 188-96.

11 Gibbons CP, Bannister JJ, Trowbridge EA, Read NW. A analysis of anal sphincter pressure and anal compliance in normal subjects. Int $\mathcal{F}$ Colorectal Dis 1986; 1: 231-7.

12 Melzak J, Porter NH. Studies on the reflex activity of the external sphincter ani in spinal man. Paraplegia 1964; 1 : 277-96.

13 Whitehead NE, Orr WC, Engel BT, Schuster MM. External anal sphincter response to rectal distension: learned resanal sphincter response to rectal distension: lea

14 Broden G, Dolk A, Frostell C, Wilsson B, Holmstrom B Broden G, Dolk A, Frostell C, Wilsson B, Holmstrom B.
Voluntary relaxation of the external anal sphincter. Dis Colon Rectum 1989; 32: 376-8. 